\title{
Invariant Subsets and Homological Properties of Orlicz Modules over Group Algebras
}

\author{
Rüya Üster and Serap Öztop*
}

\begin{abstract}
Let $G$ be a locally compact group with left Haar measure. We study the closed convex left invariant subsets of $L^{\Phi}(G)$ and characterize affine mappings from the space of nonnegative functions in $L^{1}(G)$ of norm 1 into $L^{\Phi}(G)$ spaces. We apply the results to the study of the multipliers of $L^{\Phi}(G)$. We also investigate the homological properties of $L^{\Phi}(G)$ as a Banach left $L^{1}(G)$-module such as projectivity, injectivity and flatness.
\end{abstract}

\section{Introduction}

An Orlicz space $L^{\Phi}(G)$ is a type of function space generalizing the $L^{p}$ spaces. Besides the $L^{p}$ spaces, a variety of function spaces that arise in analysis are contained naturally in Orlicz spaces. For example, $L \log L$, which is a Banach space related to Hardy-Littlewood maximal functions, and certain Sobolev spaces are contained in Orlicz spaces as subspaces. Linear properties of Orlicz spaces have been studied thoroughly (see [21], for example). Recently, Orlicz spaces and weighted Orlicz spaces over a locally compact group $G$ are considered as Banach algebras and certain cohomological properties of them are also investigated (see [1,16, 19]). However, little attention has been paid to their possible module properties (see [17], for example).

Our goal in this paper is to study possible module structures on Orlicz spaces over the group algebra of a locally compact group $G$. It is well-known that $L^{p}(G)$ is a left or right Banach $L^{1}(G)$-module (see 10 . Theorem 32.16]). In the context of Banach modules, Lau 13 studied the closed convex invariant subsets of $L^{p}(G)(1 \leq p<\infty)$; in particular, he proved that the only compact convex left or right translation-invariant subset of $L^{p}(G)$ is the origin. We obtain similar results for the $L^{\Phi}(G)$ spaces. Therefore we extend some standard results for $L^{p}(G)$ to $L^{\Phi}(G)$ spaces. Specifically, we observe that the idea of Lau can be applied to investigate the multipliers of $L^{\Phi}(G)$ spaces. Consequently we also obtain some insight into the multipliers problem for $L^{\Phi}(G)$ spaces.

Received March 24, 2019; Accepted September 11, 2019.

Communicated by Jenn-Nan Wang.

2010 Mathematics Subject Classification. Primary: 43A15, 43A22, 46E30, 46H25; Secondary: 43A20.

Key words and phrases. locally compact group, Orlicz space, invariant set, convex set, group algebra, Banach module, projectivity, injectivity, flatness, compact multiplier.

${ }^{*}$ Corresponding author. 
On the other hand, Dales and Polyakov in 6 gave a detailed study of the homological properties of modules over the group algebra of a locally compact group. By following their work, we study some homological properties of Orlicz spaces considered as $L^{1}(G)$-modules such as projectivity, injectivity and flatness.

The structure of the paper is as follows. First we introduce essential definitions and basic information that will be used in this paper. In Section 3 , we study the closed convex left-invariant subsets of $L^{\Phi}(G)$. We prove that if $G$ is locally compact but noncompact, then each closed convex left-invariant subset $C$ of $L^{\Phi}(G)$ must contain the origin. We also prove that if $C$ is compact, then $C$ contains only the origin. In Section 4, we study affine continuous mappings on subsets of Orlicz spaces. In Section 5 , we apply our results to extend some classical theorems concerning multipliers for the $L^{p}(G)$ spaces $(1 \leq p \leq \infty)$ to multipliers for the $L^{\Phi}(G)$ spaces. In particular, we extend the characterization of Brainerd and Edwards 4 from $L^{1}(G)$ to $L^{\Phi}(G)$. Finally in Section 6 , we study the projectivity of Orlicz modules over the group algebra of a locally compact group $G$ and give necessary and sufficient conditions for projectivity. We also have results on the injectivity and flatness of Orlicz modules over the group algebra of a locally compact group.

\section{Preliminaries}

In this section, we provide an overview of some basic definitions and state some technical results that will be crucial in the rest of this paper. In this paper, $G$ denotes a locally compact group with a fixed left Haar measure $\mu$.

First we recall some facts concerning Young functions and Orlicz spaces. Our main reference is [21]. A nonzero function $\Phi:[0, \infty) \rightarrow[0, \infty]$ is called a Young function if $\Phi$ is convex, $\Phi(0)=0$ and $\lim _{x \rightarrow \infty} \Phi(x)=+\infty$. For a Young function $\Phi$, its complementary function $\Psi$ is given by

$$
\Psi(y)=\sup \{x y-\Phi(x): x \geq 0\}, \quad y \geq 0 .
$$

It is easy to check that $\Psi$ is also a Young function. Also, if $\Psi$ is the complementary function of $\Phi$, then $\Phi$ is the complementary function of $\Psi$, and $(\Phi, \Psi)$ is called a complementary pair. We have the Young inequality

$$
x y \leq \Phi(x)+\Psi(y), \quad x, y \geq 0
$$

for complementary functions $\Phi$ and $\Psi$. By definition, a Young function can have the value $\infty$ at a point, and hence be discontinuous at such a point. However, we always consider a pair of complementary Young functions $(\Phi, \Psi)$ with both $\Phi$ and $\Psi$ continuous and strictly increasing. In particular, they attain positive values on $(0, \infty)$. Note that even though $\Phi$ is continuous, it may happen that $\Psi$ is not continuous. 
Let us recall some facts concerning Orlicz spaces. Suppose that $G$ is a locally compact group with a fixed Haar measure $d \mu$ and $(\Phi, \Psi)$ is a complementary Young pair. We define

$$
\mathcal{L}^{\Phi}(G)=\left\{f: G \rightarrow \mathbb{C}: f \text { is measurable and } \int_{G} \Phi(|f(x)|) d \mu(x)<\infty\right\} .
$$

Since $\mathcal{L}^{\Phi}(G)$ is not always a linear space, we define the Orlicz space $L^{\Phi}(G)$ to be

$$
L^{\Phi}(G)=\left\{f: G \rightarrow \mathbb{C}: \int_{G} \Phi(\alpha|f(x)|) d \mu(x)<\infty \text { for some } \alpha>0\right\},
$$

where $f$ indicates a $\mu$-equivalence class of measurable functions with respect to the Haar measure $d \mu$. Then the Orlicz space is a Banach space under the Orlicz norm $\|\cdot\|_{\Phi}$ defined for $f \in L^{\Phi}(G)$ by

$$
\|f\|_{\Phi}=\sup \left\{\int_{G}|f(x) \nu(x)| d \mu(x): \int_{G} \Psi(|\nu(x)|) d \mu(x) \leq 1\right\},
$$

where $\Psi$ is the complementary function to $\Phi$. One can also define the Luxemburg norm $\|\cdot\|_{\Phi}^{o}$ on $L^{\Phi}(G)$ by

$$
\|f\|_{\Phi}^{o}=\inf \left\{k>0: \int_{G} \Phi\left(\frac{|f(x)|}{k}\right) d \mu(x) \leq 1\right\} .
$$

It is known that these two norms are equivalent; that is,

$$
\|\cdot\|_{\Phi}^{o} \leq\|\cdot\|_{\Phi} \leq 2\|\cdot\|_{\Phi}^{o}
$$

and

$$
\|f\|_{\Phi} \leq 1 \quad \text { if and only if } \int_{G} \Phi(|f(x)|) d \mu(x) \leq 1 .
$$

Let us recall that a step function takes finitely many values on measurable sets of $G$. We define $M^{\Phi}(G)$ as the closure of the linear space of all step functions in $L^{\Phi}(G)$. Then $M^{\Phi}(G)$ is a Banach space and contains $C_{c}(G)$, the space of all continuous functions on $G$ with compact support, as a dense subspace [21. Proposition 3.4.3]. Moreover, $M^{\Phi}(G)^{*}$, the dual of $M^{\Phi}(G)$, can be identified with $L^{\Psi}(G)$ in a natural way 21, Theorem 4.1.6]. Another useful characterization of $M^{\Phi}(G)$ is that $f \in M^{\Phi}(G)$ if and only if for every $\alpha>0, \alpha f \in \mathcal{L}^{\Phi}(G)$ 21, Definition 3.4.2 and Proposition 3.4.3].

A Young function $\Phi$ satisfies the $\Delta_{2}$-condition if there exists a constant $K>0$ such that

$$
\Phi(2 x) \leq K \Phi(x), \quad x \geq 0 .
$$

In this case, we write $\Phi \in \Delta_{2}$. If $\Phi \in \Delta_{2}$, then it follows that $L^{\Phi}(G)=M^{\Phi}(G)$ so that $L^{\Phi}(G)^{*}=L^{\Psi}(G)$ 21, Corollary 3.4.5]. If in addition $\Psi \in \Delta_{2}$, then the Orlicz space $L^{\Phi}(G)$ is a reflexive Banach space. 
Definition 2.1. Let $\Phi_{1}$ and $\Phi_{2}$ be two Young functions. Then $\Phi_{1}$ is stronger than $\Phi_{2}$, $\Phi_{1} \succ \Phi_{2}$ (or $\Phi_{2} \prec \Phi_{1}$ ) in symbols, if

$$
\Phi_{2}(x) \leq \Phi_{1}(a x), \quad x \geq 0
$$

for some $a>0$. Further we say that $\Phi_{1}$ and $\Phi_{2}$ are strongly equivalent if $\Phi_{1} \succ \Phi_{2}$ and $\Phi_{2} \succ \Phi_{1}$. Then we write $\Phi_{1} \approx \Phi_{2}$.

We have said that Orlicz spaces are generalization of Lebesgue spaces. If we take $\Phi(x)=x^{p} / p, 1<p<\infty$, then the complementary Young function is $\Psi(y)=y^{q} / q$, with $1 / p+1 / q=1$, and the space $L^{\Phi}(G)$ becomes the Lebesgue space $L^{p}(G)$ and the norm $\|\cdot\|_{\Phi}$ is equivalent to the classical norm $\|\cdot\|_{p}$.

Our results can be applied to various Young functions (see [3, Example 8.3], 15 , Proposition 2.11] and [21, p. 15]) and concrete groups $G$ (see [8]); we point out a few below.

(1) If $\Phi(x)=x \ln (1+x)$, then $\Psi(x) \approx \cosh x-1$.

(2) If $\Phi(x)=\cosh x-1$, then $\Psi(x) \approx x \ln (1+x)$.

(3) If $\Phi(x)=e^{x}-x-1$, then $\Psi(x)=(1+x) \ln (1+x)-x$.

(4) If $\Phi(x)=x \log x(0 \leq x<\infty)$, then the Orlicz space $L^{\Phi}$ is the Zygmund space $L \log L$.

(5) If $\Phi(x)=e^{x^{\beta}}-1, x \geq 0$ with $\beta>0$, then the Orlicz space $L^{\Phi}$ is the Zygmund space $\exp L^{\beta}$.

(6) The group $\mathrm{SU}(2)$ is compact. If $f$ is an integrable function on $\mathrm{SU}(2)$, then its integral with respect to the Haar measure is

$$
\int_{\mathrm{SU}(2)} f(x) d \mu(x)=\frac{1}{2 \pi^{2}} \int_{0}^{\pi} \int_{0}^{\pi} \int_{0}^{2 \pi} f \circ \Phi(\theta, \varphi, \psi) \sin ^{2} \theta \sin \varphi d \theta d \varphi d \psi,
$$

where $\Phi$ denotes the map $(\theta, \varphi, \psi) \mapsto x=\left(x_{1}, x_{2}, x_{3}, x_{4}\right)$ in which $x_{1}=\cos \theta$, $x_{2}=\sin \theta \cos \varphi, x_{3}=\sin \theta \sin \varphi \cos \psi, x_{4}=\sin \theta \sin \varphi \sin \psi$.

(7) The group $\operatorname{GL}(n, \mathbb{R})$ is noncompact. The Haar measure on it is $|\operatorname{det} A|^{-n} d A$, where $d A$ is the Lebesgue measure on $M(n, \mathbb{R})$.

As usual, $C_{b}(G)$ is the space of bounded continuous complex-valued functions on $G$, $C_{0}(G)$ is the subspace of $C_{b}(G)$ consisting of all those functions that vanish at infinity, and $M(G)$ denotes the set of all complex regular Borel measures on $G$ which is a Banach space with respect to the norm defined by

$$
\|\mu\|=|\mu|(G)<\infty, \quad \mu \in M(G) .
$$


The convolution of $\mu \in M(G)$ and $f \in L^{\Phi}(G)$ is defined by

$$
(\mu * f)(x)=\int_{G} f\left(y^{-1} x\right) d \mu(y) .
$$

For each $a \in G$, let $\delta_{a}$ denote the measure in $M(G)$ such that $\delta_{a}(A)=1_{A}(a)$, where $1_{A}$ is the characteristic function of a subset $A$ of $G$,

$$
\left(\delta_{a} * f\right)(x)=f\left(a^{-1} x\right)=L_{a^{-1}} f(x) .
$$

Given any function $f$ on $G$, the function $\tilde{f}$ on $G$ will be defined by $\widetilde{f}(x)=f\left(x^{-1}\right)$ for each $x \in G$.

Definition 2.2. Let $X$ and $Y$ be normed spaces and $C, D$ convex subsets of $X, Y$ respectively. A map $f: C \rightarrow D$ is called affine if

$$
f(\alpha x+(1-\alpha) y)=\alpha f(x)+(1-\alpha) f(y)
$$

for all $x, y \in C$ and $\alpha \in[0,1]$.

Throughout this paper we are mainly interested in Orlicz spaces $L^{\Phi}(G)$ with the $\Delta_{2^{-}}$ condition on its Young function $\Phi$.

\section{Invariant subsets}

In this section we generalize the results of [13] concerning the characterization of closed convex invariant subsets of the $L^{p}(G)$ to the Orlicz spaces of a locally compact group $G$. Our results are motivated by the corresponding results for $L^{p}(G)$. The proof is somewhat similar to that of the $L^{p}(G)$ case, but new ideas are needed to fully adapt it to the Orlicz spaces case. We note that $L^{\Phi}(G)$ is an essential Banach $L^{1}(G)$-module with respect to the convolution [17, Lemma 3.2], it is also an $M(G)$-module. Throughout this paper we are mainly interested in the left invariant subsets of $L^{\Phi}(G)$, but similar results obtain for the right invariant subsets of $L^{\Phi}(G)$. Let us give the definition of the left invariant subsets of $L^{\Phi}(G)$.

Definition 3.1. A subset $C$ of the space $L^{\Phi}(G)$ is called left invariant if $L_{x} f \in C$ for each $f \in C$ and $x \in G$.

Note that if $f \in L^{\Phi}(G), x \in G$, then $L_{x} f \in L^{\Phi}(G)$ and $\left\|L_{x} f\right\|_{\Phi}=\|f\|_{\Phi}$ (see [17, p. 10]).

Let $K$ be a subset of $L^{\Phi}(G)$, then co $K$ will denote the convex hull of $K$. Besides the norm topology on $L^{\Phi}(G)$, we will consider the weak-topology $\omega=\sigma\left(L^{\Phi}(G), L^{\Phi}(G)^{*}\right)$, where $L^{\Phi}(G)^{*}=L^{\Psi}(G)$ and $(\Phi, \Psi)$ is a complementary pair. The weak topology on $L^{\Phi}(G)$ will be considered only occasionally, so unless otherwise specified, we will refer 
to the topological properties of sets and functions in $L^{\Phi}(G)$ with respect to the norm topology.

Further, the following subsets of $M(G)$ will be crucial in our investigation:

(1) $P(G)=\{\mu \in M(G):\|\mu\|=1$ and $\mu \geq 0\}$,

(2) $P_{1}(G)=\left\{h \in L^{1}(G):\|h\|_{1}=1\right.$ and $\left.h \geq 0\right\}$,

(3) $E(G)=\left\{\delta_{a}: a \in G\right\}$.

One can consider that $P_{1}(G)=P(G) \cap L^{1}(G)$.

The following definition and lemma appear in [13, Lemma 3.1].

Definition 3.2. Let $\nu$ denote the separated locally convex topology on $M(G)$ determined by the family of seminorms $Q=\left\{p_{f}: f \in C_{b}(G)\right\}$, such that

$$
\left|p_{f}(\mu)\right|=|\langle\mu, f\rangle|=\left|\int_{G} f d \mu\right|
$$

for each $\mu \in M(G)$. Then in the $\nu$-topology, $\mu_{\alpha} \rightarrow \mu$ means that $\int_{G} f d \mu_{\alpha} \rightarrow \int_{G} f d \mu$. We denote the weak topology of $M(G)$ by $\sigma\left(M(G), L^{\infty}(G)\right)$, while the weak ${ }^{*}$ topology by $\sigma\left(M(G), C_{0}(G)\right)$. In this way, the $\nu$-topology is $\sigma\left(M(G), C_{b}(G)\right)$. Since $C_{0}(G) \subseteq C_{b}(G) \subseteq$ $L^{\infty}(G)$, we have $\sigma\left(M(G), C_{0}(G)\right) \leq \sigma\left(M(G), C_{b}(G)\right) \leq \sigma\left(M(G), L^{\infty}(G)\right)$.

Lemma 3.3. For a locally compact group $G$, we have

$$
P(G)={\overline{P_{1}(G)}}^{\nu}=\overline{\operatorname{co} E(G)}^{\nu} .
$$

As a consequence of the Hahn-Banach separation theorem, let us note that a convex set in a Banach space which is closed in the norm topology is also closed in the weak topology.

The following lemma and theorem can be proven by adapting arguments similar to the one presented in [13, Lemma 3.2] and [13, Theorem 4.1(a)]. Therefore we omit the proofs.

Lemma 3.4. Let $G$ be a locally compact group.

(1) For each $f \in L^{\Phi}(G)$, the mapping $\mu \mapsto \mu * f$ from $M(G)$ into $L^{\Phi}(G)$ is continuous when $M(G)$ has the weak* topology and $L^{\Phi}(G)$ has the weak topology.

(2) For each $f \in L^{1}(G)$, the mapping $h \mapsto f * h$ from $L^{\Phi}(G)$ into $L^{\Phi}(G)$ is continuous when $L^{\Phi}(G)$ has the weak topology.

Theorem 3.5. Let $G$ be a locally compact group and $C$ a closed convex subset of $L^{\Phi}(G)$. Then $C$ is left invariant if and only if $h * C \subseteq C$ for each $h \in P_{1}(G)$. 
We observe the following consequence.

Corollary 3.6. Let $I$ be a closed linear subspace of $L^{\Phi}(G)$. Then $L^{1}(G) * I \subseteq I$ if and only if $I$ is a left invariant subspace of $L^{\Phi}(G)$.

Note that the set $\overline{\operatorname{co}\left\{L_{x} f: x \in G\right\}}$ is a closed, convex and left invariant subset of $L^{\Phi}(G)$. Hence, we have the following consequence.

Corollary 3.7. Let $f \in L^{\Phi}(G)$. Then

$$
\overline{\operatorname{co}\left\{L_{x} f: x \in G\right\}}=\overline{\left\{\varphi * f: \varphi \in P_{1}(G)\right\}} .
$$

Theorem 3.8. Let $G$ be a locally compact group. Then $G$ is noncompact if and only if each closed, convex, left invariant nonempty subset of $L^{\Phi}(G)$ contains the origin.

Proof. Assume that $G$ is noncompact, and take $C$ a closed, convex, left invariant nonempty subset of $L^{\Phi}(G)$. Since $G$ is noncompact for each compact subset $H$ of $G$, there exists an element $x_{H} \in G \backslash H$. It follows that the net $\left\{\delta_{x_{H}}\right\}_{H}$ weak* converges to zero in $M(G)$. Using Lemma 3.4 we conclude that the net $\left\{\delta_{x_{H}} * f\right\}_{H}$ converges to zero in the weak topology of $L^{\Phi}(G)$. The set $C$ is closed and convex, so weakly closed. Since $\left\{\delta_{x_{H}} * f\right\}_{H} \subseteq C$, then its weak limit is in $C$, that is, $C$ contains the origin.

Conversely suppose $G$ is compact. The set $C=\left\{1_{G}\right\}$ is a closed, convex, left invariant subset of $L^{\Phi}(G)$ but does not contain the origin.

Theorem 3.9. Let $G$ be a locally compact group. Then $G$ is noncompact if and only if each compact, convex, left invariant nonempty subset of $L^{\Phi}(G)$ is the origin.

Proof. Let $G$ be noncompact, $C$ a compact, convex, left invariant nonempty subset of $L^{\Phi}(G)$ and $f$ arbitrary in $C$. Let $h \in L^{\Psi}(G)$, where $\Psi$ is the complementary Young pair of $\Phi$, and consider the function $k(s)=\left\langle L_{s} f, h\right\rangle$ for $s \in G$. Then $k=f * \widetilde{h} \in C_{0}(G)$. Furthermore, $k$ is a bounded, continuous, almost periodic function on $G$. In order to justify that $k$ is almost periodic, i.e., $f * \widetilde{h} \in A P(G)$, we notice that, for each $x \in G$,

$$
\begin{aligned}
L_{x}(f * \widetilde{h})(y) & =(f * \widetilde{h})(x y)=\int_{G} f(z) \widetilde{h}\left(z^{-1} x y\right) d \mu(z)=\int_{G} f(x z) \widetilde{h}\left(z^{-1} y\right) d \mu(z) \\
& =\int_{G} L_{x} f(z) \widetilde{h}\left(z^{-1} y\right) d \mu(z)=\left(L_{x} f * \widetilde{h}\right)(y), \quad y \in G .
\end{aligned}
$$

As $\left\{L_{x} f: x \in G\right\}$ is contained in the compact set $C$, there is a net $\left\{x_{\alpha}\right\}_{\alpha} \subseteq G$ such that $\left\{L_{x_{\alpha}} f\right\}_{\alpha}$ converges in the normed space $L^{\Phi}(G)$. Since $h \in L^{\Psi}(G)$,

$$
\left\|L_{x_{\alpha}} f * \widetilde{h}-L_{x_{\beta}} f * \widetilde{h}\right\|_{\infty} \leq\left\|L_{x_{\alpha}} f-L_{x_{\beta}} f\right\|_{\Phi}\|h\|_{\Psi} \rightarrow 0
$$

so $\left\{L_{x_{\alpha}} f * \widetilde{h}\right\}_{\alpha}$ converges in $\left(C_{b}(G),\|\cdot\|_{\infty}\right)$. Since $G$ is a locally compact noncompact group, the set $A P(G) \cap C_{0}(G)$ reduces to zero, so $k \equiv 0$. Hence $f * \widetilde{h}=0$ for each 
$h \in L^{\Psi}(G)$. Now we want to show that $f=0$. Let $U_{\alpha}$ be a decreasing set of compact neighborhoods of $e$ and let $h_{\alpha}=1_{U_{\alpha}} /\left|U_{\alpha}\right|$; then $\left\|f * \widetilde{h_{\alpha}}-f\right\|_{\Phi} \rightarrow 0$. Since $h_{\alpha} \in L^{\Psi}(G)$ for each $\alpha$, it follows that $f=0$. Hence the set $C$ contains only the origin.

Conversely suppose that $G$ is compact. The set $C=\left\{1_{G}\right\}$ is a compact, convex and left invariant subset of $L^{\Phi}(G)$ but does not contain the origin.

Let us remind that more information about almost periodic functions on locally compact groups can be found in [14 for example.

\section{Affine mappings commuting with translations}

Our main aim is to determine the affine mappings and characterize the compact multipliers of Orlicz spaces. Lau 13 studied the affine mappings $T$ from a left invariant closed convex subset $A$ of $L^{q}(G)$ into a closed convex left (right) invariant subset of $L^{p}(G)$ commuting with left (right) translations. We shall study affine continuous mappings $T$ on subsets of Orlicz spaces.

The proof of the next theorem is similar to that of [13, Theorem 5.1]; we therefore omit it.

Theorem 4.1. Let $\Phi$ and $\Psi$ be Young functions and let $B$ and $C$ be closed left invariant convex subsets of $L^{\Psi}(G)$ and $L^{\Phi}(G)$, respectively. If $T: B \rightarrow C$ is continuous and affine, then the following are equivalent.

(1) $T$ commutes with left translations (i.e., $T\left(L_{x} f\right)=L_{x} T f$ whenever $x \in G$ and $f \in$ $B)$.

(2) $T(h * f)=h * T(f)$ for each $h \in P_{1}(G)$ and $f \in B$.

We have the following consequence.

Corollary 4.2. If $T: L^{\Psi}(G) \rightarrow L^{\Phi}(G)$ is a linear bounded operator, then the following are equivalent.

(1) $T\left(L_{x} f\right)=\left(L_{x} T\right) f$ whenever $x \in G$ and $f \in L^{\Psi}(G)$.

(2) $T(h * f)=h * T(f)$ for each $h \in L^{1}(G)$ and $f \in L^{\Psi}(G)$.

Theorem 4.3. Let $G$ be a locally compact noncompact group. Let $C$ be a nonempty closed convex left invariant subset of $L^{1}(G)$, and $B$ a nonempty weakly compact closed convex left invariant subset of $L^{\Phi}(G)$. If $T: B \rightarrow C$ is a continuous affine map and $T\left(L_{x} f\right)=L_{x} T f$ for each $f \in B$, then $T=0$. 
Proof. Since $B$ and $C$ are closed and convex, they are weakly closed. Since $T$ is affine and continuous, $T$ is also weakly continuous when $B$ and $C$ have their respective weak topologies. As $B$ is weakly compact, convex and $T$ is weakly continuous, $T(B)$ is a weakly compact convex left invariant subset of $L^{1}(G)$. Hence by $[13$, Theorem 4.8], $T(B)=$ $\{0\}$.

Theorem 4.4. Let $G$ be a locally compact group, $C$ be a weakly compact, closed, bounded, left invariant subset of $L^{\Phi}(G)$, and let $T: P_{1}(G) \rightarrow C$ be a continuous affine map. Then the following are equivalent.

(1) T commutes with all left translations.

(2) There exists an $f \in C$ such that $T(h)=h * f$ for $h \in P_{1}(G)$.

Proof. Let $x \in G$ and suppose that $T\left(L_{x} h\right)=L_{x} T(h)$ whenever $h \in P_{1}(G)$. By Theorem 4.1, we have $T(k * h)=k * T(h)$ for $k, h \in P_{1}(G)$. Suppose that $\left\{U_{\alpha}\right\} \subseteq P_{1}(G)$ is a bounded approximate identity for $L^{1}(G)$. Since $T\left(U_{\alpha}\right) \in C$ is bounded and $C$ is weakly compact, there is an $f \in C$ such that $\left\{T\left(U_{\alpha}\right)\right\}_{\alpha}$ converges to $f$ in the weak topology. Then

$$
T(h)=\lim _{\alpha} T\left(h * U_{\alpha}\right)=\lim _{\alpha} h * T\left(U_{\alpha}\right)=h * f .
$$

Conversely let $x \in G$ and suppose that there is $f \in C$ such that $T(h)=h * f$ for all $h \in P_{1}(G)$. Then

$$
L_{x} T(h)=L_{x}(h * f)=\delta_{x^{-1}} *(h * f)=\left(\delta_{x^{-1}} * h\right) * f=L_{x} h * f=T\left(L_{x} h\right) .
$$

This completes the proof.

\section{Compact multipliers}

A multiplier is an important concept for Banach spaces. We want to study the problem of multipliers on $L^{\Phi}$ spaces. We apply our results in Section 3 to extend some classical theorems concerning multipliers for the $L^{p}$ spaces, $1 \leq p<\infty$, to multipliers for $L^{\Phi}$ spaces of a locally compact group. For $p=1$ and $G$ is a locally compact group, the space of multipliers is precisely $M(G)$. This is a famous result of Wendel 24]. For a locally compact noncompact group, Sakai 23 proved that a compact multiplier on $L^{1}(G)$ commuting with left (right) translations is identically zero. On the other hand Akemann [2] proved that if $G$ is a compact group, then $M\left(L^{1}(G), L^{1}(G)\right)$ consists only of compact operators, where $M\left(L^{1}(G), L^{1}(G)\right)$ denotes the set of all bounded linear operators on $L^{1}(G)$ that commute with the left (right) translations.

Our main purpose is to obtain some results on the compact multipliers problem for Orlicz spaces. We characterize the compact multipliers of $L^{\Phi}(G)$ by taking $L^{\Phi}(G)$ as an 
$L^{1}(G)$-module. We are able to extend these results to compact multipliers from $L^{1}(G)$ to $L^{\Phi}(G)$.

Let us start with the definition of the left multipliers of $L^{\Phi}(G)$.

Definition 5.1. A bounded linear operator $T$ from $L^{\Psi}(G)$ to $L^{\Phi}(G)$ is called a left multiplier for the pair $\left(L^{\Psi}(G), L^{\Phi}(G)\right)$ if $T\left(L_{x} f\right)=L_{x}(T f)$ for all $f \in L^{\Psi}(G)$ and $x \in G$. We denote the set of left multipliers for the pair $\left(L^{\Psi}(G), L^{\Phi}(G)\right)$ by $M\left(L^{\Psi}(G), L^{\Phi}(G)\right)$.

For the following theorem we assume that both $\Phi$ and $\Psi$ are Young functions satisfying the $\Delta_{2}$-condition.

Theorem 5.2. Let $G$ be a locally compact group. For a bounded linear operator $T$ from $L^{1}(G)$ to $L^{\Phi}(G)$, the following are equivalent.

(1) $T \in M\left(L^{1}(G), L^{\Phi}(G)\right)$.

(2) There exists an $f \in L^{\Phi}(G)$ such that $T(h)=h * f$ for $h \in L^{1}(G)$.

Proof. Suppose first that $T \in M\left(L^{1}(G), L^{\Phi}(G)\right)$. Let $\left\{u_{\alpha}\right\}_{\alpha}$ be an approximate identity for $L^{1}(G)$ such that $\left\|u_{\alpha}\right\|_{1}=1$ for all $\alpha$. Then for all $h \in L^{1}(G)$ we have $\left\|h * u_{\alpha}-h\right\|_{1} \rightarrow 0$. It follows that $\left\{T\left(h * u_{\alpha}\right)\right\}_{\alpha}$ converges to $T(h)$ in the norm topology for all $h \in L^{1}(G)$. On the other hand, the net $\left\{T\left(u_{\alpha}\right)\right\}_{\alpha}$ is bounded in the reflexive space $L^{\Phi}(G)$, so passing to a subnet if necessary, we may assume that $\left\{T\left(u_{\alpha}\right)\right\}_{\alpha}$ converges in the weak topology to some $f \in L^{\Phi}(G)$. Then using Corollary 4.2 and Lemma 3.4, we have

$$
T(h)=\lim _{\alpha} T\left(h * u_{\alpha}\right)=\lim _{\alpha} h * T\left(u_{\alpha}\right)=h * f .
$$

The proof of the converse part is similar to the proof of the corresponding part of Theorem 4.4 .

Theorem 5.3. Let $G$ be a compact group. Every multiplier $T$ from $L^{1}(G)$ into $L^{\Phi}(G)$ is compact.

Proof. Let $T \in M\left(L^{1}(G), L^{\Phi}(G)\right)$. By Theorem 5.2 there exists $f \in L^{\Phi}(G)$ such that $T(h)=h * f$, for all $h \in L^{1}(G)$. We will prove that $T$ is a compact operator, i.e., $T$ takes the unit ball of $L^{1}(G)$ into a relatively compact set of $L^{\Phi}(G)$. As $G$ is compact and the mapping $L_{x} f$ is continuous, the set $\overline{\operatorname{co}\left\{L_{x} f: x \in G\right\}} \|^{\|\cdot\|_{\Phi}} \subseteq L^{\Phi}(G)$ is compact, so using Corollary 3.7 it follows that $\left\{\varphi * f: \varphi \in P_{1}(G)\right\}$ is relatively compact in $L^{\Phi}(G)$. If $h \in L^{1}(G)$ and $\|h\|_{1} \leq 1$, then $h=\left(h_{1}-h_{2}\right)+i\left(h_{3}-h_{4}\right)$, where $h_{j}>0$ and $\left\|h_{j}\right\|_{1}<1$, $j=1,2,3,4$. It follows that $T\left(h_{j}\right)$ is in $C=\{\lambda \varphi * f: \lambda \in[0,1]\}$. Since $C$ is relatively compact, it follows that $T(h)$ lies in the relatively compact set $(C-C)+i(C-C)$. Hence $T$ is a compact operator. 
Theorem 5.4. For a locally compact noncompact group $G$, the only compact multiplier $T$ from $L^{\Psi}(G)$ into $L^{\Phi}(G)$ is zero.

Proof. Let $T$ be a compact operator and $T L_{x}=L_{x} T$. Then, the closure of the set $\left\{T(f):\|f\|_{\Psi} \leq 1\right\}$ is a compact, convex, left invariant, nonempty subset of $L^{\Phi}(G)$, so by Theorem 3.9, it reduces to zero. Hence $T=0$.

The following consequence is apparent from Theorem 4.3 .

Theorem 5.5. Let $G$ be a locally compact noncompact group. Let $A$ be a weakly compact closed left invariant subspace of $L^{\Phi}(G)$. If $T$ is a bounded linear operator from $A$ into $L^{1}(G)$ commuting with left translations, then $T$ is zero.

\section{Projectivity, injectivity and flatness}

Homological properties of $L^{p}(G)$ as an $L^{1}(G)$-module have been studied by Dales and Polyakov [6]. In this section we characterize some homological properties of $L^{\Phi}(G)$ considered as $L^{1}(G)$-modules, such as projectivity, injectivity and flatness.

First we recall some homological properties of a Banach algebra. Let $A$ be a Banach algebra and $E$ be a Banach left $A$-module. We denote by $A$ - $\bmod$ and $\bmod -A$ the categories of Banach left $A$-modules and of Banach right $A$-modules, respectively. These classes of modules are defined in [5, 0.3.2] and [9, Definition 2.6.1]. Suppose that $E \in A$-mod. Set

$$
A E=\operatorname{span}\{a x: a \in A, x \in E\} .
$$

Then $E$ is called essential if $\overline{A E}=E$, where the overbar denotes closure. In the case $A$ has a bounded approximate identity $\left\{e_{\alpha}\right\}_{\alpha}, E$ is essential if and only if $\lim _{\alpha} e_{\alpha} x=x$ for all $x \in E$ (see [7, Corollary 15.3]).

We denote by $B(E, F)$ the Banach space of all bounded linear operators from $E$ to $F$. We call a $T \in B(E, F)$ admissible if there exists $S \in B(F, E)$ with $T \circ S=\mathrm{id}_{F}$. If $E, F \in A$-mod, then ${ }_{A} B(E, F)$ denotes the closed linear subspace of $B(E, F)$ consisting of all left $A$-module morphisms. An operator $T \in{ }_{A} B(E, F)$ is called a retraction if there exists $S \in{ }_{A} B(F, E)$ with $T \circ S=\mathrm{id}_{F}$. Similar definitions apply when $E, F \in \bmod -A$.

Definition 6.1. Let $A$ be a Banach algebra, and let $P \in A$-mod. Then $P$ is called a projective $A$-module if for each $E, F \in A$-mod, for each admissible epimorphism $T \in$ ${ }_{A} B(E, F)$ and for each $S \in{ }_{A} B(P, F)$, there exists $R \in{ }_{A} B(P, E)$ such that $T \circ R=S$.

Definition 6.2. Let $A$ be a Banach algebra and $J \in A$-mod. Then $J$ is called injective if for each $E, F \in A$-mod, each admissible monomorphism $T \in{ }_{A} B(E, F)$ and each $S \in$ ${ }_{A} B(E, J)$, there exists $R \in{ }_{A} B(F, J)$ such that $R \circ T=S$. 
Definition 6.3. Let $A$ be a Banach algebra, and let $E \in A$-mod. Then $E$ is flat if the dual $E^{*}$ of $E$ is injective in $\bmod -A$.

It is known that $L^{1}(G)$ has the approximation property by [22, Example 4.5] and $\widehat{\otimes}$ denotes the projective tensor product of any Banach spaces.

We investigate the homological properties of $L^{\Phi}(G)$ induced by the structure of the underlying group. We will use [6, Proposition 1.2(i)] for the following theorem.

Theorem 6.4. Let $G$ be a locally compact group and $\Phi$ be a Young function. Then $L^{\Phi}(G)$ is a projective $L^{1}(G)$-module if and only if $G$ is compact.

Proof. First suppose that $G$ is compact. It is known that if $G$ is compact, then every essential Banach left $L^{1}(G)$-module is projective $[20,1.2]$. Therefore $L^{\Phi}(G)$ is a projective $L^{1}(G)$-module.

For the converse, suppose on the contrary that $G$ is not compact. Let $V$ and $W$ be compact symmetric neighborhoods of the identity element $e_{G}$ such that $V^{2} \subseteq W$. Let $f(x)=\frac{\chi_{V} * \chi_{V}(x)}{\mu(V)}=\frac{1}{\mu(V)} \int_{G} \chi_{V}(y) \chi_{V}\left(y^{-1} x\right) d \mu(y)$ for $x \in G$. Then $f \in C_{c}(G), f(G) \in$ $[0,1]$, with $f\left(e_{G}\right)=1, \operatorname{supp}(f) \subseteq V,\|f\|_{\infty}=1$ and $\|f\|_{1} \leq 1$. It is clear that $f * f \neq 0$ and $\operatorname{supp}(f * f) \subseteq W$. Since $L^{1}(G)$ has the approximation property by [22, Example 4.5] and also $L^{\Phi}(G)$ is essential by [17, Lemma 3.2], then by [6, Proposition 1.2] there exists a $T \in{ }_{L^{1}(G)} B\left(L^{\Phi}(G), L^{1}(G)\right)$ such that $T(f * f) \neq 0$.

Set $\eta=\|f * T(f)\|_{1} / 2$ and fix $k \in \mathbb{N}$. Choose a function $g \in C_{c}(G)$ with support $K_{1}$ such that $\|T(f)-g\|_{1}<1 / k$ and $\|f *(T(f)-g)\|_{1}<\eta$. It follows that $\|f * g\|_{1}>\eta$.

Since $G$ is compact, there exist $s_{1}, s_{2}, \ldots, s_{k} \in G$ such that the sets $s_{j}(V \cup K)^{2}$ are pairwise disjoint for $j=1,2, \ldots, k$. Indeed, set $s_{1}=e$ and consider the set $s_{1}(V \cup$ $K)^{2}(V \cup K)^{-2}$ which is a compact subset of $G$. Since $G$ is not compact, there exists $s_{2} \in G \backslash s_{1}(V \cup K)^{2}(V \cup K)^{-2}$. This way one can choose inductively each $s_{j} \in G \backslash$ $\bigcup_{i=1}^{j-1} s_{i}(V \cup K)^{2}(V \cup K)^{-2}$.

Set $f_{j}=s_{j} * f$ and $\lambda=\sum_{j=1}^{k} f_{j} * f$. Since $s_{1} V, \ldots, s_{k} V$ are pairwise disjoint, we have $\left\|\sum_{j=1}^{k} f_{j}\right\|_{\infty}=1$ and $\left\|\sum_{j=1}^{k} f_{j} * g\right\|_{1}=k\|f * g\|_{1}$. Then

$$
\|T(\lambda)\|_{1}=\left\|\sum_{j=1}^{k} f_{j} * T(f)\right\|_{1} \geq\left\|\sum_{j=1}^{k} f_{j} * g\right\|_{1}-1=k\|f * g\|_{1}-1>k \eta-1 .
$$

On the other hand,

$$
\begin{aligned}
\left\|\sum_{j=1}^{k} f_{j} * f\right\|_{\Phi} & =\sup \left\{\int_{G}\left|\sum_{j=1}^{k} f_{j} * f(x) h(x)\right| d \mu(x): \int_{G} \Psi(|h(x)|) d \mu(x) \leq 1\right\} \\
& \leq \sup \left\{\int_{G} \sum_{j=1}^{k}\left|f_{j} * f(x) h(x)\right| d \mu(x): \int_{G} \Psi(|h(x)|) d \mu(x) \leq 1\right\}
\end{aligned}
$$




$$
\begin{aligned}
& =\sup \left\{\sum_{j=1}^{k} \int_{G}\left|f * f(x) h\left(s_{j} x\right)\right| d \mu(x): \int_{G} \Psi(|h(x)|) d \mu(x) \leq 1\right\} \\
& =k\|f * f\|_{\Phi} .
\end{aligned}
$$

We obtain $\left\|\sum_{j=1}^{k} f_{j} * f\right\|_{\Phi} \leq k\|f * f\|_{\Phi}$. Since $f \in C_{c}(G)$, it follows that $f * f \in L^{\Phi}(G)$ and $\|\lambda\|_{\Phi} \leq k\|f * f\|_{\Phi}$.

Thus

$$
k \eta-1 \leq\|T(\lambda)\|_{1} \leq\|T\|\|\lambda\|_{\Phi} \leq k\|T\|\|f * f\|_{\Phi} .
$$

Consequently,

$$
k \eta \leq k\|T\|\|f * f\|_{\Phi}+1 .
$$

This holds for each $k \in \mathbb{N}$, which is the required contradiction. Therefore $G$ must be compact, as claimed.

Remark 6.5. For the "if" part we can also use the result of Racher 20, Proposition 1.4] related to the projectivity of Banach modules. It is known that Orlicz space is separable if and only if $G$ is separable (see [21, Theorem 3.5.1]).

Observation 6.6. Let $G$ be a locally compact group, $(\Phi, \Psi)$ complementary pair and $\Psi \in \Delta_{2}$. If $L^{\Phi}(G)$ is a projective $L^{1}(G)$-module with $G$ being separable (second countable) then $G$ is compact.

Now we seek to determine when the module $L^{\Phi}(G)$ is injective and flat. Let us recall that if $E \in A$-mod, then $E^{*} \in \bmod -A$ is the dual module of $E$ with the module operation specified by the formula

$$
\langle x, \lambda a\rangle=\langle a x, \lambda\rangle, \quad a \in A, x \in E, \lambda \in E^{*} .
$$

Similarly $E^{*} \in A$-mod when $E \in \bmod -A$ and in this case the module operation in $E^{*}$ is specified by

$$
\langle x, a \lambda\rangle=\langle x a, \lambda\rangle, \quad a \in A, x \in E, \lambda \in E^{*} .
$$

We know that every module (left or right) over an amenable algebra is flat and every dual module is injective [9, VII.2.29]. Moreover, Johnson proved that the Banach algebra $L^{1}(G)$ is amenable if and only if the group $G$ is amenable [11, Theorem 2.5]. If we combine these results we have the following observations.

Remark 6.7. Let $\Phi$ be a Young function. If $G$ is an amenable group, then

(1) $L^{\Phi}(G)$ is a flat Banach left $L^{1}(G)$-module,

(2) $L^{\Psi}(G)$, the dual module of $L^{\Phi}(G)$, is an injective Banach right $L^{1}(G)$-module,

(3) if $\Phi, \Psi \in \Delta_{2}$, then since $L^{\Phi}(G)$ is a dual module, $L^{\Phi}(G)$ is both an injective and flat Banach left $L^{1}(G)$-module. 


\section{Acknowledgments}

This study was funded by Scientific Research Projects Coordination Unit of İstanbul University, project number 29526.

We are grateful to an anonymous referee for a careful reading of the manuscript and for helpful comments.

\section{References}

[1] I. Akbarbaglu and S. Maghsoudi, Banach-Orlicz algebras on a locally compact group, Mediterr. J. Math. 10 (2013), no. 4, 1937-1947.

[2] C. A. Akemann, Some mapping properties of the group algebras of a compact group, Pacific J. Math. 22 (1967), 1-8.

[3] C. Bennett and R. Sharpley, Interpolation of Operators, Pure and Applied Mathematics 129, Academic Press, London, 1988.

[4] B. Brainerd and R. E. Edwards, Linear operators which commute with translations I: Representation theorems, J. Austral. Math. Soc. 6 (1966), 289-327.

[5] H. G. Dales, Banach Algebras and Automatic Continuity, London Mathematical Society Monographs, New Series, 24, Oxford University Press, New York, 2000.

[6] H. G. Dales and M. E. Polyakov, Homological properties of modules over group algebras, Proc. London Math. Soc. (3) 89 (2004), no. 2, 390-426.

[7] R. S. Doran and J. Wichmann, Approximate Identities and Factorization in Banach Modules, Lecture Notes in Mathematics 768, Springer-Verlag, Berlin, 1979.

[8] J. Faraut, Analysis on Lie Groups: An introduction, Cambridge Studies in Advanced Mathematics 110, Cambridge University Press, Cambridge, 2008.

[9] A. Ya. Helemskii, The Homology of Banach and Topological Algebras, Mathematics and its Applications (Soviet Series) 41, Kluwer Academic Publishers, Dordrecht, 1989.

[10] E. Hewitt and K. A. Ross, Abstract Harmonic Analysis II: Structure and analysis for compact groups analysis on locally compact abelian groups, Springer-Verlag, Berlin, 1997.

[11] B. E. Johnson, Cohomology in Banach Algebras, Memoirs of the American Mathematical Society 127, American Mathematical Society, Providence, R.I., 1972. 
[12] R. Larsen, An Introduction to the Theory of Multipliers, Die Grundlehren der mathematischen Wissenschaften, Band 175, Springer-Verlag, Heidelberg, 1971.

[13] A. T. M. Lau, Closed convex invariant subsets of $L_{p}(G)$, Trans. Amer. Math. Soc. 232 (1977), 131-142.

[14] A. T. M. Lau and V. Losert, Complementation of certain subspaces of $L_{\infty}(G)$ of a locally compact group, Pacific J. Math. 141 (1990), no. 2, 295-310.

[15] W. A. Majewski and L. E. Labuschagne, On applications of Orlicz spaces to statistical physics, Ann. Henri Poincaré 15 (2014), no. 6, 1197-1221.

[16] A. Osançliol and S. Öztop, Weighted Orlicz algebras on locally compact groups, J. Aust. Math. Soc. 99 (2015), no. 3, 399-414.

[17] S. Öztop and E. Samei, Twisted Orlicz algebras I, Studia Math. 236 (2017), no. 3, 271-296.

[18] _ Twisted Orlicz algebras II, Math. Nachr. 292 (2019), no. 5, 1122-1136.

[19] S. Öztop, E. Samei and V. Shepelska, Weak amenability of weighted Orlicz algebras, Arch. Math. (Basel) 110 (2018), no. 4, 363-376.

[20] G. Racher, On the projectivity and flatness of some group modules, in: Banach Algebras 2009, 315-325, Banach Center Publications 91, Polish Acad. Sci. Inst. Math., Warsaw, 2010.

[21] M. M. Rao and Z. D. Ren, Theory of Orlicz Spaces, Marcel Dekker, New York, 1991.

[22] R. A. Ryan, Introduction to Tensor Products of Banach Spaces, Springer Monographs in Mathematics, Springer-Verlag London, London, 2002.

[23] S. Sakai, Weakly compact operators on operator algebras, Pacific J. Math. 14 (1964), 659-664.

[24] J. G. Wendel, Left centralizers and isomorphisms of group algebras, Pacific J. Math. 2 (1952), 251-261.

Rüya Üster and Serap Öztop

Department of Mathematics, Faculty of Science, İstanbul University, İstanbul, Turkey

E-mail address: ruya.uster@istanbul.edu.tr, oztops@istanbul.edu.tr 